regarding IL23 (276 vs $262 \mathrm{pg} / \mathrm{ml}, \mathrm{p}>0.4$ ) and IL17 levels (184 vs $233 \mathrm{pg} / \mathrm{ml}$, $p>0.2)$. Only $22(6.6 \%)$ AS patients carried the protective rs 11209026 A allele, while $206(61.7 \%)$ carried the rs $11209032 \mathrm{~A}$ risk allele $(p=0.03)$. There was no demonstrable influence of individual genotypes (A vs $G, A A$ vs $A G$ vs $G G$ ) or haplotypic combinations on BASFI, spinal function tests, CRP, ESR, IL-23 or IL-17 levels (all $p>0.3$ )

Conclusions: While there is a high prevalence of the IL-23R rs11209032 A risk allele in Caucasian AS patients, this has no demonstrable bearing on clinical disease measures or serum IL-23 and IL-17 levels.

Acknowledgements: The authors wish to acknowledge the technical assistance by mrs K Nilsen and the financial support of the North Norwegian Health Authority Research Fund

Disclosure of Interest: None declared

DOI: 10.1136/annrheumdis-2017-eular.5882

\section{FRI0427 FIRST DESCRIPTION OF GAMMA DELTA T CELLS AT NORMAL HUMAN ENTHESIS}

R.J. Cuthbert ${ }^{1}$, E. Fragkakis ${ }^{2}$, R. Dunsmuir ${ }^{2}$, P. Giannoudis ${ }^{1}$, E. Jones ${ }^{1}$, D. McGonagle ${ }^{1}$. ${ }^{1}$ Leeds Institute of Rheumatic and Musculoskeletal Medicine, University of Leeds; ${ }^{2}$ Department of Spinal surgery, The Leeds teaching Hospitals NHS Trust, Leeds, United Kingdom

Background: Recent animal studies have suggested that $\gamma \delta \mathrm{T}$-cells accumulate at enthesis, secrete IL-17 and are responsible for driving the spondyoarthritis (SpA) phenotype resulting from IL-23 overexpression in mice $(1,2)$. In humans examination of the immunological profile of enthesis has been hampered by lack of tissue. Recently, we used a novel strategy to show that group 3 innate lymphoid cells are present at the human enthesis (3). Here we extend our methodology to examine the broader immunological profile of human enthesis and to determine if $\gamma \delta \mathrm{T}$-cells are also present.

Objectives: To characterise $\gamma \delta$ T-cells at human enthesis and adjacent perientheseal bone

Methods: Human etheseal soft tissue (EST) and peri-entheseal bone (PEB) was harvested from normal spinous process in patients undergoing elective spinal orthopaedic procedures. Interspinous EST was dissected from PEB and enzymatically digested, followed by isolation of mononuclear cells. Flow cytometry was then used to determine the proportion of B-cells (CD45+, CD19+) NK cells (CD45+, CD3-, CD56+) and T-cells (CD45+, CD3+). T-cells were then sub divided based on expression of CD4 (T-helper cells), CD8 (Cytotoxic T-cells) and TCR $\gamma \delta$ $(\gamma \delta \mathrm{T}-\mathrm{cellls})$. All entheseal data was compared to age-matched peripheral blood from healthy controls.

Results: Entheseal digests contained on average a lower proportion of T-cells compared to peripheral blood $(\mathrm{p}=0.018)$. However, the proportion of T-cells not expressing either CD4 or CD8 was greater in entheseal tissues $(p=0.021)$, this population was largely composed of $\gamma \delta$ T-cells. As a proportion of T-cells $\gamma \delta$ T-cells were 6 fold more numerous in EST compared to peripheral blood $(p=0.024)$, and PEB had 3 fold more. $37 \%$ of EST $\gamma \delta$ T-cells expressed CCR6 this compared to $26 \%$ and $34 \%$ in PEB and peripheral blood respectively.

Conclusions: $\gamma \delta$ T-cells are present in normal human enthesis and $\gamma \delta \mathrm{T}$-cells constitute a greater proportion of the T-cell pool compared to peripheral blood, making it likely that they represent a tissue resident population. Additionally, we observed a very similar proportion $\gamma \delta \mathrm{T}$-cells that expressed CCR6, a functional marker for IL-17 production, as was observed in mice (2). This is the first description of $\gamma \delta$ T-cells at the human enthesis and offers tentative confirmation of findings in mouse models where these cells play a key role in SpA pathogenesis.

\section{References:}

[1] Sherlock JP, Joyce-Shaikh B, Turner SP, Chao CC, Sathe M, Grein J, et al. IL23 induces spondyloarthropathy by acting on ROR-gammat+ CD3+CD4-CD8entheseal resident T cells. Nat Med. 2012 Jul;18(7):1069-76. PubMed PMID: 22772566.

[2] Reinhardt A, Yevsa T, Worbs T, Lienenklaus S, Sandrock I, Oberdörfer L, et al. IL-23-dependent $\gamma \delta \mathrm{T}$ cells produce IL-17 and accumulate in enthesis, aortic valve, and ciliary body. Arthritis \& Rheumatology. 2016.

[3] Cuthbert R, Fragkakis E, Dunsmuir R, Millner P, El-Sherbiny Y, McGonagle D. AB0028 Innate Lymphoid Cells Are Present at Normal Human Enthesis Providing A Potential Mechanism for Spondyloarthropathy Pathogenesis. Annals of the Rheumatic Diseases. 2016;75(Suppl 2):906-.

Disclosure of Interest: None declared

DOI: 10.1136/annrheumdis-2017-eular.6421

\section{FRI0428 THE JAK1-SELECTIVE INHIBITOR, FILGOTINIB, INHIBITS INFLAMMATION PATHWAYS OBSERVED IN AN IL23-INDUCED PSORIATIC ARTHRITIS MOUSE MODEL}

R. Blanqué ${ }^{1}$, M. Ongenaert ${ }^{2}$, C. David ${ }^{1}$, C. Robin-Jagerschmidt ${ }^{1}$, A. Cauvin ${ }^{1}$, C. Saccomani ${ }^{1}$, P. Clement-Lacroix ${ }^{1}$, S. Dupont ${ }^{1}$, R. Galien ${ }^{1}$. ${ }^{1}$ Galapagos SASU, Romainville, France; ${ }^{2}$ Galapagos NV, Mechelen, Belgium

Background: Psoriatic arthritis (PsA) is a heterogeneous chronic inflammatory disease characterized by musculoskeletal involvement and extra-skeletal manifestations such as psoriasis, uveitis and Inflammatory Bowel Disease (IBD). The importance of several pro-inflammatory cytokines, in addition to TNFa, IL-12 and IL-23 which are targets of current treatments, suggests that novel therapies may benefit patients. The JAKs (a family of 4 non-receptor tyrosine kinases) are crucial for the signaling of many pro-inflammatory cytokines. In this regard, the JAK1-selective inhibitor filgotinib (GLPG0634, GS-6034) has shown efficacy in patients with rheumatoid arthritis (RA), a disease that shares some hallmarks with PsA, as well as in Crohn's disease (CD), making this molecule a potential therapy for PsA.

Objectives: To gain insight into filgotinib mode of action using a PsA preclinical model by analysing the gene expression signature of filgotinib in mouse phalanges and colon tissues from an IL23-induced PsA mouse model.

Methods: Spondyloarthopathy was induced by hydrodynamic injection of IL-23 enhanced Episomal Expression Vector (EEV). Animals were treated with filgotinib or vehicle from day 10 (therapeutic mode) and sacrificed after 16 days of treatment. RNAs were extracted from the phalanges and the proximal colon, and transcriptome assays were performed using the Agilent SurePrint G3 mouse chip. Data analysis was performed using empirical Bayes methods and linear models (limma BioConductor package).

Results: In mice, IL-23 induced changes in the transcriptome in both phalanges and colon that were marked by effects on genes related to the IL-23/T 17 axis. Microarray analysis performed on mouse phalanges and colon revealed that filgotinib partially reversed the impact of IL-23 on gene expression in colon and in phalanges. In both tissues, filgotinib signature was different but some impacted biological programs were similar. A consistent interferon signature was counteracted by filgotinib in both tissues with decreased expression of common genes such as Apobec3, Gbp8, ligp1 and Oas3. Several markers of inflammation or associated with IL-23 activitywere also decreased with common on Kynu and Cd96 gene expression in both tissues. Of interest, filgotinib repression on inflammatory gene expression was stronger in colon compared to phalanges (IL1b, Clec4e). Moreover expression of some genes involved in gut homeostasis that were induced by IL-23 were decreased by filgotinib in the colon, notably Fpr2 (receptor for formyl peptides) and Mmp7. In phalanges, gene expression associated with IL-23-induced disease was also reversed by filgotinib treatment. II19, Mtcl1 and TIr1 which are key mediators in psoriasis, or Rankl that is involved in bone remodeling in PsA were differently regulated by IL-23 and filgotinib. Conclusions: Systemic expression of IL-23 in mice generated a PsA phenotype that was associated with altered gene expression in diseased tissues. A strong interferon signature was reversed by filgotinib as were several inflammation and disease markers. Together with the previous Phase 2 clinical results in RA and $C D$, these data support the study of filgotinib for the treatment of PsA patients.

Disclosure of Interest: R. Blanqué Employee of: Galapagos SASU, M. Ongenaert Employee of: Galapagos NV, C. David Employee of: Galapagos SASU, C. RobinJagerschmidt Employee of: Galapagos SASU, A. Cauvin Employee of: Galapagos SASU, C. Saccomani Employee of: Galapagos SASU, P. Clement-Lacroix Employee of: Galapagos SASU, S. Dupont Employee of: Galapagos SASU, R. Galien Employee of: Galapagos SASU

DOI: 10.1136/annrheumdis-2017-eular.4911

\section{FRI0429 DYSREGULATION OF THE SPLICING MACHINERY IN LEUKOCYTES FROM ANKYLOSING SPONDYLITIS PATIENTS IS ASSOCIATED TO DISEASE PATHOGENESIS}

Y. Jimenez-Gomez ${ }^{1}$, C. Lopez-Pedrera ${ }^{1}$, S. Pedraza-Arévalo ${ }^{2}$, M. del Río-Moreno $^{2}$, M.C. Ábalos-Aguilera ${ }^{1}$, P. Ruiz-Limon ${ }^{1}$, C. Perez-Sanchez ${ }^{1}$ M.C. Castro ${ }^{1}$, N. Barbarroja ${ }^{1}$, I. Arias-de la Rosa ${ }^{1}$, P. Font ${ }^{1}$, A. Escudero ${ }^{1}$, J.P. Castaño ${ }^{2}$, R.M. Luque ${ }^{2}$, E. Collantes-Estevez ${ }^{1}{ }^{1}{ }^{1}$ IMIBIC/Reina Sofia University Hospital/Cordoba University; ${ }^{2}$ MIBIC/Reina Sofia University Hospital/Cordoba University; CIBERobn and ceiA3, Cordoba, Spain

Background: Ankylosing spondylitis (AS) is a chronic inflammatory disease, of unknown aetiology, associated to the development of several comorbidities such as atherosclerosis. Splicing is a post-transcriptional process involved in the RNA maturation. Recent studies have revealed that a pathological dysregulation of the splicing machinery or spliceosome is associated to several human diseases. Yet, the spliceosome alterations have not been described in AS.

Objectives: 1) To analyze whether dysregulation of the spliceosome is present in AS. 2) To evaluate the association between the alteration of this process and the clinical, inflammatory, oxidative, and atherogenic profiles present in this pathology. Methods: Fourteen AS patients and 14 healthy donors (HDs) were included in the study. Disease function and activity status were analyzed using the BASFI and BASDAI. The expression of selected components of the major- $(n=12)$ and minor-spliceosome $(n=4)$, and splicing factors $(n=28)$ was evaluated in purified monocytes, lymphocytes and neutrophils from patients and HDs ( $n=14$ each) by Fluidigm methodology. Oxidative stress, inflammation and atherogenesis were evaluated by flow cytometry and RT-PCR. Endothelial function was determined by the post occlusive hyperaemia test using Laser-Doppler.

Results: Compared to HDs, a significant dysregulation in the expression of relevant splicing factors and spliceosome components was found in all the leukocyte subtypes from AS patients, being neutrophils which displayed higher number of altered molecules. Interestingly, a specific altered profile of major- and minor-spliceosome members, and splicing factors was observed when compared lymphocytes (U4, U6, NOVA1, RMB17), monocytes (PRP8, SF3B TV2, CELF4, ESRP2, RBM3, SAM68 TV1, SRSF10, TIA1) and neutrophils (U11, U2, U2AF2, U11, CA 150, ESRP1, PSF, PTB, SRM160). 